delivery, intrauterine growth restriction (IUGR) and preeclampsia. Conceiving in periods of low disease activity helps to reduce these complications.

Objectives: This project aims to describe the occurrence of pregnancy complications among women with immune-mediated rheumatic diseases and to study the associated clinical factors.

Methods: A retrospective longitudinal study was performed including consecutive pregnant women with immune-mediated rheumatic diseases seen in a multidisciplinary group for autoimmune diseases during pregnancy, in a tertiary hospital, between January 2010 and December 2015. Clinical and demographic data, as well as and pregnancy outcomes, were collected through consultation of clinical files. The factors associated with pregnancy manifestations (premature delivery, flares during pregnancy, recurrent pregnancy loss and foetal growth restriction) were studied using Mann-Whitney, qui-square and fisher tests (SPSS 24.0). Significance level was set as $<0.05$.

Results: We included 151 gestations from a total of 140 women with a mean age of $32,5 \pm 4,4$ years; 4 gestations were twin pregnancies. Within these 151 gestations, $54(35,8 \%)$ women had SLE, $17(11,3 \%)$ had Sjögren's syndrome, 17 $(11,3 \%)$ had rheumatoid arthritis, 41 had APS $(27,2 \%), 11(7,3 \%)$ had Behcet's disease, $4(2,6 \%)$ had systemic sclerosis, $8(5,3 \%)$ had mixed connective tissue disease and $16(10,6 \%)$ had other immune-mediated diseases. $35(23,2 \%)$ had anti-SSA/La antibodies, $18(11,9 \%)$ had anti-SSB antibodies, $6(4,0 \%)$ had anti-URNP antibodies and $43(28,5 \%)$ had anti-nuclear antibodies. Seven $(4,6 \%)$ of the women developed gestational diabetes and $4(2,6 \%)$ developed gestational hypertension. Furthermore, $54(35,8 \%)$ women had had previous miscarriages. Prematurity occurred more frequently among neonates with IUGR $(53.8 \%$ vs $46.1 \% ; p=0.04)$, and was associated with gestational diabetes $(21.4 \%$ vs $2.9 \%$; $\mathrm{p}=0.018$ ). It also occurred more frequently in multiple pregnancies $(75 \%$ vs $16.4 \%$; $p=0.001)$, mothers taking glucocorticoids $(28.6 \%$ vs $9.2 \% ; p=0.003)$ and active rheumatic disease at conception ( $23 \%$ vs $6.8 \% \mathrm{p}=0.03$ ). No statistically significant differences were observed in the occurrence of different pregnancy complications among different diseases or in presence of different antibodies.

Conclusions: Our study proved a link between immune-mediated rheumatic diseases and specific pregnancy outcomes such as prematurity and IUGR. Outcomes were worse when taking glucocorticoids, when gestational diabetes were developed and when conception occurred in a period of active disease.

Disclosure of Interest: None declared

DOI: 10.1136/annrheumdis-2017-eular.6095

\section{AB0145 TLR4 SIGNALING PATHWAY MEDIATES THE SENESCENCE OF BONE MARROW- MESENCHYMAL STEM CELLS FROM SYSTEMIC LUPUS ERYTHEMATOSUS PATIENTS}

J. Ji, Y. Wu, X. Kong, Z. Zhang, Z. Gu. Department of Rheumatology, Affiliated Hospital of Nantong University, Nantong, China

Background: Previous studies of our research group revealed the senescence of bone marrow-mesenchymal stem cells from systemic lupus erythematosus patients, which participated in the development of SLE. "Inflammatory microenvironment" played a very important role in cellular senescence. In the preliminary experiments, we discovered the level of HMGB1 in serum and Peripheral blood mononuclear cells from SLE patients was higher than those of The healthy control group.

Objectives: The aim of this study was to investigate whether HMGB-1 can lead to senescence BM-MSCs from SLE patients and its possible mechanism.

Methods: Tewelve female SLE patients and healthy subjects were enrolled in the study. All patients were females, and their age distribution was similar to that of the cases. All BM-MSCs were Isolated by density gradient centrifugation. Western Blotting and immunofluorescence were used to distinguish the difference of expression and localization of TLR4 signaling pathway between normal group and SLE group. Different concentrations $(0.01,0.1,1,10 \mathrm{ug} / \mathrm{ml})$ of HMGB-1 (the endogenous ligand of TLR4) stimulated normal BM-MSCs, then detecting expression of TLR4 signal by WB, observing the activity of $\beta$-gal of cells, the changes of cytoskeletal structure by F-actin staining and the distribution of cell cycle by flow cytometry. We used small interfering RNA (siRNA) to interfere the expression of TLR4.

Results: BM-MSCs from SLE patients showed prominent features of senescence, characterized by impaired capacities of proliferation, increased SA- $\beta$-gal activity, and disordered cytoskeleton distribution, and abnormal activation of TLR4 signaling transduction, high level of phosphorylated $\mathrm{p} 65$. І $_{\kappa} \mathrm{B} \alpha$. After stimulation of HMGB1 in normal MSCs, TLR4 signaling was activated. And, the cell volume and the number of SA- $\beta$-gal positive in SLE BM-MSCs was increased. The organization of cytoskeleton was neatly disordered. The rate of cell proliferation was decreased. The inhibitors of HMGB-1 and small interfering RNA (siRNA) of TLR4 can significantly reverse the senescence.

Conclusions: HMGB-1 binded to TLR4, and by activating MyD88/IRAK/TRAF pathway, promoted NF-кB signal transduction, thereby affected the expression of cell cycle-related proteins, and then resulted in senescence of MSCs from SLE patients.

Acknowledgements: This research was supported by grants from the National Natural Science Foundation of China (81471603).

Disclosure of Interest: None declared

DOI: 10.1136/annrheumdis-2017-eular.5375

\section{AB0146 INVOLVEMENT OF PERIPHERAL CD8 T CELL SUBSETS IN SYSTEMIC LUPUS ERYTHEMATOSUS}

J. Kikuchi $^{1}$, M. Ushikubo ${ }^{1,2}$, S. Saito ${ }^{1}$, K. Yamaoka ${ }^{1}$, K. Sugahara ${ }^{3}$, K. Chiba ${ }^{3}$, T. Takeuchi ${ }^{1}$. ${ }^{1}$ Division of Rheumatology, Department of Internal Medicine, Keio University School of Medicine; ${ }^{2}$ Department of Connective Tissue Diseases, National Tokyo Medical Center, Tokyo; ${ }^{3}$ Sohyaku. Innovative Research Division, Mitsubishi Tanabe Pharma Corporation, Kanagawa, Japan

Background: Although the mainstream of pathogenesis of systemic lupus erythematosus (SLE) is thought to be interactions between antigen-presenting cells like dendritic cells, helper T cells, B cells and cytokines, previous reports suggested CD8 T cells also involve in the pathogenesis of SLE ${ }^{1,2}$. However the associations between subsets of CD8 T cells and clinical manifestations remains unclear.

Objectives: We conducted standard immunophenotyping analysis with peripheral blood from SLE patients and focused on CD8 T cell subsets to elucidate the association with clinical phenotype and serological markers.

Methods: Peripheral blood was obtained from inactive SLE patients and healthy subjects as controls and also from active SLE before and 3 months after treatment. CD8 $T$ cell subsets were measured by flow cytometry with fresh whole blood samples.

Results: Thirty-four active SLE patients and 38 inactive patients and 22 healthy controls (HCs) whose age and sex were matched with those in SLE patients were enrolled. Mean SLE disease activity index (SLEDAI) was 14.2 and 1.8 in active and inactive patients, respectively. Among CD8 T cell subsets, the proportion of HLA-DR+ cells was significantly higher in SLE patients than HCs and positively correlated with SLEDAI $(p=0.016$, rho $=0.283)$, and was also higher in patients with nephritis than patients without nephritis $(p=0.074)$, though it did not reach statistical significance. The proportion of naive CD8 T cells positively correlated with the titer of anti-dsDNA antibody $(p=0.011$, rho $=0.30)$ and $\mathrm{C} 1 \mathrm{q}$ immune complex levels $(\mathrm{p}=0.043$, rho $=0.25$ ), and negatively correlated with serum complement levels $(p=0.019$, rho $=-0.34)$. The proportion of central memory CD8 T cells $(T c m)$ negatively correlated with SLEDAI $(p<0.001$, rho $=-0.43)$, the titer of dsDNA antibody $(p<0.001$, rho $=-0.51)$ and $C 1 q$ immune complex levels $(p<0.001$, rho $=-0.44)$, and positively correlated with serum complement levels $(p<0.001$, rho $=0.49)$, and was lower in the patients with nephritis $(p=0.041)$, skin rash $(p<0.001)$, and fever $(p=0.002)$ than the patients without them. The proportion of effector memory CD8 T cells (Tem) was lower in SLE and negatively correlated with the titer of anti-dsDNA antibody $(p=0.028$, rho $=$ -0.26 ). Sixteen active patients were treated with prednisolone (PSL) $50 \mathrm{mg}$ (mean) with concomitant immunosuppressant; eight were treated with cyclophosphamide. Mean SLEDAI decreased from 15.9 to 3.9 at 3 months after the treatments. The proportion of naïve CD8 T cells significantly decreased $(p=0.003)$, and the proportions of HLA-DR+ cells and Tem increased at 3 months $(p=0.011$ and $p=0.007$, respectively). The proportion of Tem increased and negatively correlated with SLEDAI only with those who were treated with cyclophosphamide.

Conclusions: Pathological state of SLE positively correlated with the proportion of naïve CD8 T cells and negatively correlated with the proportion of Tem and these subsets were affected by treatment with PSL and immunosuppressant particularly cyclophosphamide. These results indicate that CD8 T cells are involved in pathophysiology and could potentially become a biomarker or a treatment target in SLE patients.

References:

[1] Blanco P, et al. Arthritis Rheum 2005; 52: 201-11.

[2] Kim JS, et al. Rheumatology (Oxford) 2012; 51: 1587-94.

Disclosure of Interest: J. Kikuchi: None declared, M. Ushikubo: None declared, S. Saito: None declared, K. Yamaoka: None declared, K. Sugahara Employee of: Mitsubishi Tanabe Pharma Corporation, K. Chiba Employee of: Mitsubishi Tanabe Pharma Corporation, T. Takeuchi: None declared

DOI: 10.1136/annrheumdis-2017-eular.4948

\section{AB0147 THE EFFECT OF TREATMENT WITH HYDROXYCHLOROQUINE ON SOLUBLE TISSUE FACTOR LEVELS IN PATIENTS WITH ANTIPHOSPHOLIPID ANTIBODIES AND ANTIPHOSPHOLIPID SYNDROME WITH AND WITHOUT UNDERLYING SYSTEMIC LUPUS ERYTHEMATOSUS}

K. Schreiber ${ }^{1,2}$, K. Breen ${ }^{1}$, K. Parmar ${ }^{1}$, B.J. Hunt ${ }^{1} .{ }^{1}$ Centre for Thrombosis and Haemostasis, Guy's and St Thomas' NHS Foundation Trust, London, United Kingdom; ${ }^{2}$ Department of Rheumatology, Copenhagen University Hospital, Copenhagen, Denmark

Background: Antiphospholipid syndrome (APS) is characterized by venous, microvascular and/or arterial and/or obstetric morbidity (obstetric APS) in patients who are persistently positive for antiphospholipid antibodies (aPL)[1]. The mainstay of treatment is based on anticoagulation therapy; however, increasing interest is currently received by the antimalarial hydroxychloroquine (HCQ). The use of HCQ has been associated with a reduced risk of thrombosis but HCQ's antithrombotic mechanism of action is unclear particularly in patients with aPL and APS.

Objectives: The aim of our study was to assess soluble tissue factor (TF) levels in HCQ naïve-patients with persistent aPL or APS at baseline and 12 weeks after commencing $\mathrm{HCQ}$. We hypothesise that $\mathrm{HCQ}$ lowers levels of soluble TF. 